\title{
Heat capacity of cylindrically confined helium: theoretical predictions versus experimental data
}

\author{
K.A. Chalyy \\ Faculty of Physics, Kiev Taras Shevchenko National University, Acad. Glushkov Pr., 6, Kiev 03022, Ukraine \\ E-mail: kirchal@univ.kiev.ua
}

Received November 25, 2003, revised February 19, 2004

\begin{abstract}
Different systems which could exhibit size-dependent second-order phase transitions have been studied experimentally during the last quarter century. The validity of the proposed theoretical results is verified by comparing high-resolution experimental data with an analytical evaluation of the heat capacity of confined ${ }^{4} \mathrm{He}$. It is shown that the theoretical approach to the problem of the finite-size effect gives results that reasonably match the experimental data over a wide range of system sizes, from tens of nanometers up to a few micrometers for the cylindrical type of confinement geometry. The dependences of the shift of transition temperature on the cylinder size and boundary conditions are analyzed. The agreement of the results with finite-size scaling theory is confirmed.
\end{abstract}

PACS: 65.20.+w, 67.40.Kh, 68.35.Rh

\section{Introduction: confined helium experiments}

The physical properties of a liquid systems are strongly dependent on its size. Spatial limitation of a liquid matter system can cause significant changes in its properties. Analysis of such microscopic systems and their thermodynamic, static properties as well as measurement of the optical and transport properties has become a key task in various fields. The behavior of condensed and soft matter systems at small length scales is becoming a topic of technological importance [1] in view of modern achievements in micro- and nanofabrication techniques [2].

Most of the recent advances toward better understanding of the effect of spatial limitation and finite-size induced phenomena could not be realized without basic research results of confined helium experiments. Among the pioneering investigations in this field was an experimental study by Chen and Gasparini [3], which was conducted about quarter century ago. Chen and Gasparini measured the specific heat of ${ }^{4} \mathrm{He}$ near the superfluid transition for films from about $10 \AA$ to $56 \AA$ thick and for samples confined to a cylindrical geometry of 200 to $2000 \AA$ in diameter.

Comparatively recently, the most reliable data $[1,4,5]$ on cylindrically confined helium have became available from the Stanford research group lead by J.A. Lipa. It was pointed out in [4] that the common confining geometries are planes and cylinders, allowing the study of crossover from bulk three-dimensional behavior to two- and one-dimensional behavior, respectively. By approaching the transition very closely, it becomes possible to use well-defined geometries amenable to detailed theoretical analysis. When the correlation length $\xi$ is on the order of the average distance to the walls, the sharp peak in the specific heat of bulk helium is predicted to be rounded and shifted to a lower temperature. However, in the region where the confinement effects are strong, there does not appear to be a detailed theoretical prediction of the shape of the heat capacity curve that is directly applicable to cylindrical geometry [6].

In [6] Coleman and Lipa reported the results of the heat capacity measurements as a function of temperature near the $\lambda$ point of ${ }^{4} \mathrm{He}$ confined in 8 - $\mu$ m diameter cylinders that have exceptionally uniform diameters and smooth surfaces. Later in [1] the results of a more specific analysis of the $8-\mu \mathrm{m}$ data were presented and the new data of an experimental study of a $0.26-\mu \mathrm{m}$ cylinder were discussed. In contrast to the much earlier results [3] with smaller holes, they found good agreement with scaling-law exponents derived by renormalization methods. 
For comparably large systems the size-dependent shift of the critical temperature is extremely small, which makes it difficult to detect due to the smearing effect of Earth's gravity. Up to this date most experiments on the effects of confinement on helium in planar and cylindrical geometries have been restricted to the submicron (or nano-) regime $[3,7,8]$. However, Lipa et al. [1] stressed that using high-resolution thermometry techniques [9], it has become possible to explore the region extending up to about $100 \mu \mathrm{m}$ under microgravity conditions. This circumstance provides sufficient background to consider the comparably big systems as «finite-size» one. This development has dramatically increased the range over which length scaling can be tested and has eased the problem of uncontrolled surface effects.

Experimental studies in finite-size liquids are quite difficult to conduct due to the fact that blurring effect of the Earth's gravity often causes phenomena very similar to the finite-size effect. This creates a high demand for a theory that fits the existing experimental results and which could be used for explicit calculations of the properties in a wide range of sizes and for different types of system geometries. At present, for the case of cylindrical geometry, the primary source of predictions is the Monte Carlo studies (see, for example, [10]).

In this paper, we present a comparative study of experimental data and theoretical approaches to the finite-size effect in the liquid helium heat capacity in the close vicinity of the $\lambda$-transition temperature $T_{\lambda}$. Actual analytical calculations are conducted without taking into account the gravity effect. Consequently, it could be applicable for reliable comparison with the results of microgravity experiments in Earth orbit or with a number of Earth-based measurements of the ${ }^{4} \mathrm{He}$ heat capacity in cylindrical confinement. In such Earth-based measurements the characteristic size of the system is supposed to be small enough to allow one to neglect the influence of gravity on the precision of new transition temperature detection.

\section{Helium-in-cylinrical-confinment heat capacity: theoretical predictions}

A system can be considered as finite-sized near the critical point or the phase transition point if its characteristic linear size $Z$ becomes comparable with the correlation length $\zeta$ of the order parameter fluctuation. As is well known from the fluctuation (scaling) theory of phase transitions (see, e.g., [11-13]), the correlation length is expressed by

$$
\zeta=\zeta_{\mathrm{o}} \tau^{-v}
$$

where the amplitude of the correlation length in classical liquids can reach the value $\zeta_{0} \approx 1-10 \mathrm{~nm}$, and $\tau=\left|T-T_{c}\right| / T_{C}$ is the temperature variable. Nowadays, it has become possible to approach the critical point of classical liquids with respect to the temperature variable to within «distances» like $\left|T-T_{c}\right|_{\min } \approx$ $\approx 10^{-2}-10^{-3} \mathrm{~K}$ for $T_{c} \approx 10^{2} \mathrm{~K}$, or, using dimensionless values, $\tau_{\min }=\left|T-T_{c}\right|^{\prime} / T_{c} \approx 10^{-5}$. As the critical exponent of the correlation length is equal to $v \approx 0.6705$ [14] for the space dimensionality $d=3$, the maximum values of the correlation length in classical liquids can approach such values as $\zeta \approx 1-10 \mathrm{~nm} \times$ $\times\left(10^{-5}\right)^{-0,67} \approx 10^{3}-10^{4} \mathrm{~nm}$.

Spatial insufficiency on an even bigger scale can be realized for quantum liquids, where, because of the very small values of the critical temperature, the correlation length becomes two orders more; i.e., for instance, in liquid helium $\tau \approx 10^{-8}$ and $\zeta / \zeta_{0} \approx 10^{5.4}$. So, classical liquid systems that have linear size $Z$ up to a micrometer (for quantum liquids, even to tens of micrometers) could be considered as finite-sized near the critical point (or $\lambda$ point). The physical properties of finite-size systems preserve their singular behavior only for the directions in which the system still has semi-infinite size. However, taking into account the limited temperature resolution of even extremely precise modern experiments [4,7], it should be noted that over the limit of a system's smallest confining size of about a few hundreds micrometers the finite-size effect seems to be almost impossible to detect even under conditions of microgravitation. In the case of Earthbased experiments due to the gravity effect that limit will not exceed ten micrometers [15].

Using high-resolution thermometers [9] it has become possible to determine the exact location of the $\lambda$-transition temperature with a precision of $\pm 5 n \mathrm{~K}$ [6]. Since the useful resolution of measurements at the lambda point on Earth is limited in principle by the pressure dependence of the transition temperature (gravity effect), it is important to be sure that the observed rounding of the specific heat is in fact produced by finite-size effects. In spite of that, it has been suggested [6] that gravity does not play a major role in the actual Earth-based experiments with the typical confined size less than $10 \mu \mathrm{m}$. This statement is supported by the fundamental studies of Ahlers (see, for example, [16]) and could be explained in terms of direct calculations of temperature variation due to the gravity effect. In order to proceed, we have to consider the limiting value of the cylinder length $L$ over which the results of a study of the finite-size effect become independent of further increase of $L$. It was found $[17,18]$ that there is no need to take the actual $L \rightarrow \infty$ limit, because it turns out that for $L=5 D$ and 
larger ( $D$ is the diameter of a pore), the finite-size effect due to the finite value of $L$ becomes insignificant. The vertical variation of transition temperature $\delta T_{\lambda}$ over height $L$ of the sample due to the hydrostatic pressure induced by Earth's gravity is determined [19] by the expression $\delta T_{\lambda}=\gamma L$ with $\gamma=1.273 \mu \mathrm{K} / \mathrm{cm}$. Thus, for cylinder of radius $D=8 \mu \mathrm{m}[1,6]$ even the enhanced limit of $L=80 \mu \mathrm{m}$ corresponds to a maximum variation $\delta T_{\lambda} \approx 10^{-8} \mathrm{~K}$ between the bottom and top of the chamber. It is assumed [20] that data are unaffected by gravity if $\left|T-T_{\lambda}\right| \geq 10 \delta T_{\lambda}$. In all cases to be discussed below this condition is satisfied. This makes it possible to neglect the gravity effect and simultaneously conduct a reliable study of the finitesize effect at some range of confining sizes. The corresponding theoretical predictions could be suitable for comparison with actual Earth-based measurements $[3,5,6]$. Furthermore, the same theoretical approach is applicable for comparative study of the results of microgravity Confined Helium Experiment (CHEX) [1] conducted aboard the space shuttle and, hopefully, future experiments $[17,20]$ which are scheduled to be carried out on the Low Temperature Microgravity Physics Facility (LTMPF) aboard the International Space Station.

The major interest in the statistical physics approach to finite-size phase transitions is to find the pair correlation function $G_{2}$ of the order parameter fluctuations and the associated correlation length $\zeta$. We consider here a cylindrical sample with diameter $D$ and length $L>>D$ and study the region where the value of the correlation length $\zeta$ becomes comparable to or even larger than $D$ but still smaller than $L$. The correlation length could be defined [21] as $\zeta=$ $=\left(M_{2}\right)^{1 / 2}$ in terms of the pair correlation function $G_{2}$ of the order parameter fluctuations for the reduced geometry of cylindrical form, where $M_{2}$ denotes the normalized second moment of $G_{2}$ (see for details [22]). The pair correlation function associated with the order parameter fluctuations was deduced by applying the Helmholtz operator. We have derived $G_{2}$ from the corresponding differential equation with Dirichlet boundary conditions on the inner wall surface of a cylinder and the condition of $G_{2}$ decay at long distances, i.e., zero value of $G_{2}$ on the top and bottom ends of the cylinder. In particular, this type of boundary condition seems to represent the boundary conditions of the real confined helium experiments more appropriately [23,24]. As follows from the discussion in [22] anomalous growth of the correlation length might occur at some new temperature $T_{c}^{*}$ which is lower than the bulk $T_{c}$ and accordingly is located in the region of negative $\tau$. For such a case the behavior of the correla- tion length might obey a scaling law akin to (1), namely

$$
\zeta^{*}=\zeta_{0} \tau^{*-v},
$$

where $\tau^{*}=\left(T-T_{c}^{*}\right) / T_{c}^{*}$ is the new temperature variable for a spatially limited system. It is necessary to notice that the defining condition (2) used above means physically that upon the achievement of the new critical temperature $T_{c}^{*}$ of a spatially limited liquid in the sample of cylindrical geometry there might exist anomalous growth of the longitudinal component of the correlation length $\zeta^{*}$ along the cylinder axis as is described by

$$
\zeta^{*}=\zeta_{0}\left[\tau+\left(\frac{\psi_{1}}{K}\right)^{1 / \nu}(\tau+1)\right]^{-v},
$$

where $\zeta_{0}$ is the amplitude of correlation length, $K=$ $=r / \zeta_{0}$ is the geometrical factor, $r$ is the cylinder radius, $\tau=\left(T-T_{c}\right) / T_{c}$ is the temperature variable, $v$ is the critical exponent of the correlation length, and the quantity $\psi_{1}$ is determined by the expression $J_{0}\left(\psi_{1}\right)=e A$ for the case of the constant boundary condition $G_{2}=A$ on the cylinder wall surface. Here $J_{0}$ is the zero-order Bessel function, and $e$ is a transcendental constant equal to 2.7182 . In the case of the zero boundary condition $A=0$, the value of the parameter $\psi_{1}$ is equal to the first zero of the zero-order Bessel function, $\mu_{1}=2.4048$. Here, the boundary effect to the pair correlation function $G_{2}$ is translated to the boundary condition for the corresponding differential equation [22].

In order to verify the validity of the theoretical results proposed here, and before in [22,25], it is important to have the possibility of comparing the results with high-resolution experimental data. Since most of the data $[1,3,5,6]$ available up to this date are derived from the study of the heat capacity of confined ${ }^{4} \mathrm{He}$, it is necessary to obtain an analytical expression particularly for this property. Such calculations could be conducted on a background of the formulas for the temperature dependence of the correlation length and well-known scaling relations for the heat capacity. While the correlation length $\zeta$ is proportional to $\tau^{-v}$, the analogous formula for the temperature dependence of the heat capacity $C$ reads $C \sim \tau^{-\alpha}$, where $\alpha$ is the heat capacity critical exponent, which assumed positive. Combining it [26], one can get the scaling relation between the heat capacity and the correlation length:

$$
C \sim \zeta^{\alpha / \nu} .
$$

The hyperscaling relation $\alpha=2-3 v$ [27] with $v=$ $=0.6705$ yields $\alpha / v=-0.0172$. In fact, near the 
lambda point a small negative value of $\alpha \approx-0.026$ was observed in early experiments [28]. Later, in the most precise microgravity experiment in Earth orbit [29], it was confirmed to be $\alpha=-0.01285$. However, it is known that near the liquid-gas critical point the exponent $\alpha$ appears to be positive and close to 0.1 [30].

Using the general formula (4) it became possible to obtain a formula for the heat capacity in a particular type of confining geometry by assuming $\zeta=\zeta^{*}$. The correlation length $\zeta^{*}$ expressed by Eq.(3) is directly associated with the cylindrical geometry and corresponds to the case of zero boundary condition for the pair correlation function $G_{2}$ on the inner surface of the confining system. That is why the new expressions for the heat capacity also will retain special parameters, which are related to particular form of the system. This makes possible an easy comparison with the data of the corresponding experiments. For the case of cylindrical geometry it reads

$$
C_{\mathrm{cyl}} \propto\left[\tau+\left(2 \frac{\mu_{1}}{K_{\mathrm{cyl}}}\right)^{1 / v}(\tau+1)\right]^{-\alpha} .
$$

This equation (5) shows that the heat capacity in the cylindrical geometry remains finite at the bulk $T_{\lambda}$ $(\tau=0)$ and grows up to its limit at some point below $T_{\lambda}$ in the region of negative $\tau$.

To carry out further comparison with experimental data, here in Eq.(5) the geometrical factor was redefined: $K_{\text {cyl }}=D / \zeta_{0}$, with $D$ being the cylinder diameter. The amplitude of correlation length $\zeta_{0}$ for helium is equal to $0.36 \mathrm{~nm}$ below and $0.143 \mathrm{~nm}$ above the transition temperature [31]. The parameter $\mu_{1}=2.4048$ is the first zero of the cylindrical Bessel function [32]. The critical exponent $v$ is taken to be equal to 0.6705 [14] and $\alpha=-0.01285$ [29].

Using Eq. (5) it is possible to evaluate the shift $\Delta \tau$ of the transition temperature from the location of the heat capacity maximum. The corresponding new transition temperature $T_{c}^{*}(K)$ is determined by the expression

$$
T_{c}^{*}(K)=T_{c}\left[1+\left(2 \mu_{1} / K_{\text {cyl }}\right)^{1 / \nu}\right]^{-1} .
$$

In Table the experimental data from Refs. 1,3,5,6 for confined helium are combined with the results calculated from Eq. (5) for the same values of the system's sizes. Here, in Table $\Delta \tau_{E}=\left(T_{\lambda}-T_{m}\right) / T_{m}$ represents the shifts of the helium heat capacity maximum $T_{m}$ from its bulk value $T_{\lambda}$ observed experimentally in respect that $T_{\lambda}>T_{m}$, and $\Delta \tau_{T}$ is the shift calculated according to the proposed theoretical approach from the expression:

$$
\Delta \tau_{T}=\left(2 \mu_{1} \zeta_{0} / D\right)^{1 / \nu} .
$$

Transition temperature shift: Experimental data versus correspondent theoretical calculation for the helium confined in cylindrical samples of different diameters.

\begin{tabular}{c|c|c|c}
\hline \hline $\begin{array}{c}\text { Diameter } D \\
\text { of the cylinder }\end{array}$ & $\begin{array}{c}\text { Shift } \Delta \tau_{E}, \\
\text { Experiment }\end{array}$ & $\begin{array}{c}\text { Shift } \Delta \tau_{T}, \\
\text { Theory }\end{array}$ & $\begin{array}{c}\text { Point's } \\
\text { number } / \text { [Ref.] }\end{array}$ \\
\hline \hline $300 \AA$ & $4.0 \cdot 10^{-3}$ & $3.6 \cdot 10^{-3}$ & $C 1 /[3]$ \\
$800 \AA$ & $6.5 \cdot 10^{-4}$ & $8.3 \cdot 10^{-4}$ & $C 2 /[3]$ \\
$1000 \AA$ & $4.3 \cdot 10^{-4}$ & $5.9 \cdot 10^{-4}$ & $C 3 /[3]$ \\
$2000 \AA$ & $1.2 \cdot 10^{-4}$ & $2.1 \cdot 10^{-4}$ & $C 4 /[3]$ \\
$0.26 \mu \mathrm{m}$ & $1.53 \cdot 10^{-4}$ & $1.44 \cdot 10^{-4}$ & $C 5 /[1,5]$ \\
$8 \mu \mathrm{m}$ & $1.20 \cdot 10^{-6}$ & $0.87 \cdot 10^{-6}$ & $C 6 /[6]$ \\
$8.17 \mu \mathrm{m}$ & $1.24 \cdot 10^{-6}$ & $0.85 \cdot 10^{-6}$ & $C 7 /[1,5]$ \\
\hline \hline
\end{tabular}

\section{Discussion}

In Fig. 1 the dependence of the shift of the transition temperature $\Delta \tau_{T}$ on the cylindrical pore diameter $D$, which varies from $10 \mathrm{~nm}$ up to $10 \mu \mathrm{m}$, is presented in $\log -\log$ scale for the case of the zero boundary condition. It shows that the value of $\Delta \tau_{T}$ is decreasing with respect to cylinder diameter growth. The slope of the plot in Fig. 1 is $1 / v \approx 1.49$, in agreement with the finite-size scaling theory predictions [13,33]: $\Delta \tau=$ $=a H^{-1 / v}$, where $a$ is a constant depending on the geometry. In the case considered above, the linear size of the system $H$ is treated as the cylinder diameter $D$, and consequently the expression for the scaling coefficient $a$ reads: $a=\left(2 \mu_{1} \zeta_{0}\right)^{1 / v}$.

In Table the point set $\Delta \tau_{E} C 1-C 4$ is taken from the graph, presented in the pioneering paper [3]. At that

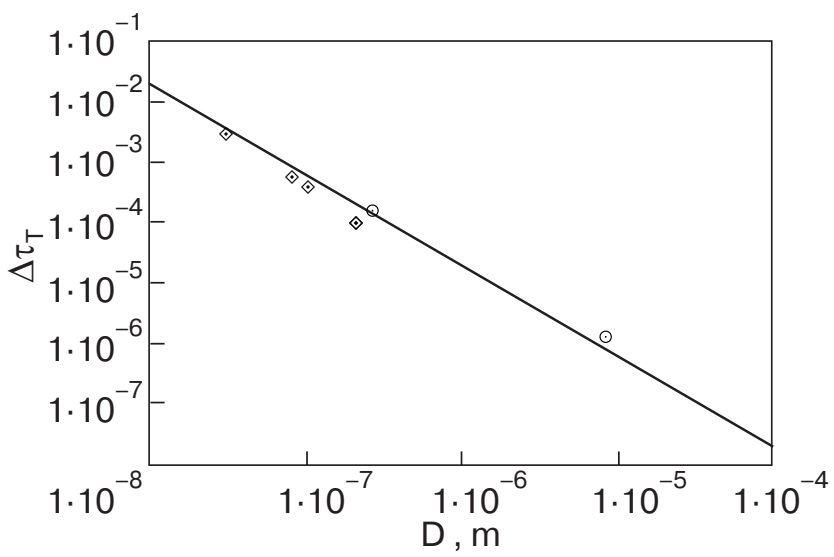

Fig. 1. The dependence of the shift of the transition temperature $\Delta \tau_{T}$ on the cylindrical pore diameter $D$ in $\log -\log$ scale (according to Eq.(7)) - solid line. The slope of the plot corresponds to $v=0.6705$. $\diamond$ represents the experimental data of Chen and Gasparini [3]. $\odot$ represents the experimental data of Lipa et al. [5]. 
time the cylindrical pores with diameters 300,800 , 1000 , and $2000 \AA$ were investigated. Since it was difficult to determine $\Delta \tau_{E}$ precisely from that graph in the paper [3] (tabulated data were not included there), the accuracy of these data is limited. A comparison shows that the theoretical values $\Delta \tau_{T}$ systematically overestimate the shift of the new transition temperature by $30 \%$ on average, except $C 1$, with a better match. It should be noticed that points $C 1-C 4$ were taken in experiments conducted about twenty five years ago, but their results are still cited in the related modern papers (see, for example, [6]). However, some remarks [5] concerning the low accuracy and rough agreement with scaling of these early results have to be considered. Values $\Delta \tau_{T}$ are calculated for the case of zero boundary condition for the pair correlation function $G_{2}$ that provides the maximum possible value of the shift $\Delta \tau$ for a particular size of the system. However, in a real experiment, depending on the specific features of the surface interaction, the value of the boundary constant could be nonzero - as is also expected to be the case for the binary-mixture experiments - which lead to a decrease of the shift of the new transition temperature. This could be one of the possible reasons of this deviation $\Delta \tau_{E}$ with $\Delta \tau_{T}$.

Judicious consideration of the boundary condition is important. As we deal with a liquid in a reduced geometry of cylindrical form, here we introduce the boundary condition to the pair correlation function $G_{2}$ on the inner surface of the cylinder in the form $G_{2}=A$. Here $A$ is a certain constant, the exact value of which depends on particular problem. This expression applies to the limiting case of completely hydrophobic inner cylinder surface for $A=0$ (i.e., the zero boundary condition of the first type sometimes referred as the «homogeneous condition») and hydrophilic one for $A=1$. In practice the situation could appear with intermediate values of $A$, indicating a partial wetting for which the numerical value in the function varies from 0 to 1 . In the general case of constant boundary condition $G_{2}=A$ on the cylinder surface the shifts $\Delta \tau_{T}$ will depend on $A$ as

$$
J_{0}\left(D \Delta \tau_{T}^{v} / 2 \zeta_{0}\right)=e A
$$

For example, for the helium sample in a cylindrical chamber of diameter $1000 \AA$ (this value corresponds to the point $C 3$ in Table) in the case of the constant boundary condition, the dependence of the shift of the transition temperature $\Delta \tau_{T}$ on the boundary constant $A$ is illustrated by Fig. 2. As can be seen from Fig. 2, while the boundary constant $A$ is decreasing in value the shift $\Delta \tau_{T}$ grows and reaches its maximum at $A=0$. Since $A$ characterizes the border of the system, this behavior of the shift of the transition temperature may

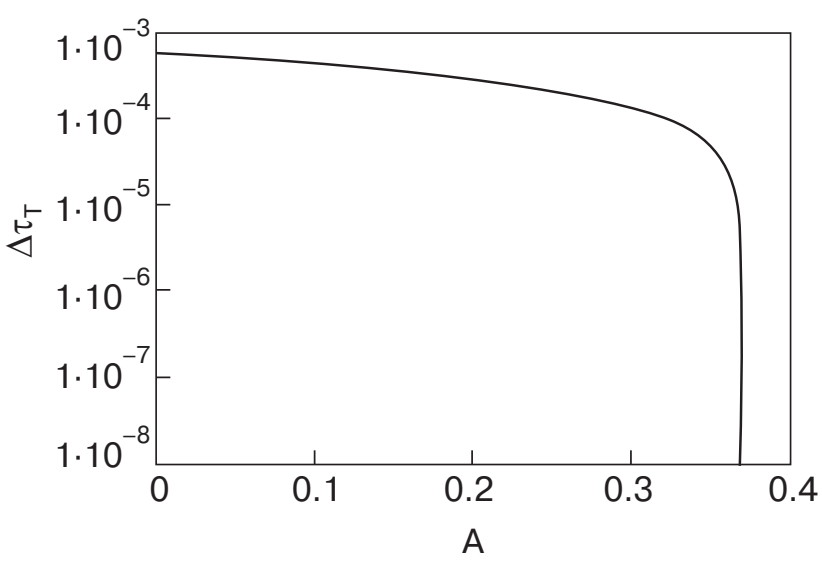

Fig. 2. The dependence of the shift of the transition temperature $\Delta \tau_{T}$ on the boundary constant $A$ for the sample in a cylindrical pore of diameter $1000 \AA$ in semilog scale (according to Eq.(8)).

be explained as being due to a competition between the surface interaction near the walls and finite-size effects.

Concerning point $C 6$ for $8 \mu \mathrm{m}$ cylinder [6], the theoretical value $\Delta \tau_{T}$ appears to be about $30 \%$ smaller than the results of that high-precision modern experiment. Reference points $C 5$ and $C 7$ are taken from the most recent measurements of the heat capacity of $8.17 \mu \mathrm{m}$ and $0.26 \mu \mathrm{m}$ cylindrically confined helium that are available now $[1,5]$. This set of the points shows better agreement between the theoretical calculations and experimental data in terms of the shift of transition temperature, especially for the $0.26 \mu \mathrm{m} \mathrm{di}-$ ameter pore. It was noticed [5] that the results of the new measurement for $8.17 \mu \mathrm{m}$ cylinders do not compare well with previous observations [6] in the region near the heat capacity peak. In contrast, the results for $0.26 \mu \mathrm{m}$ Anopore cylinders are in good agreement with Monte Carlo predictions [10,18].

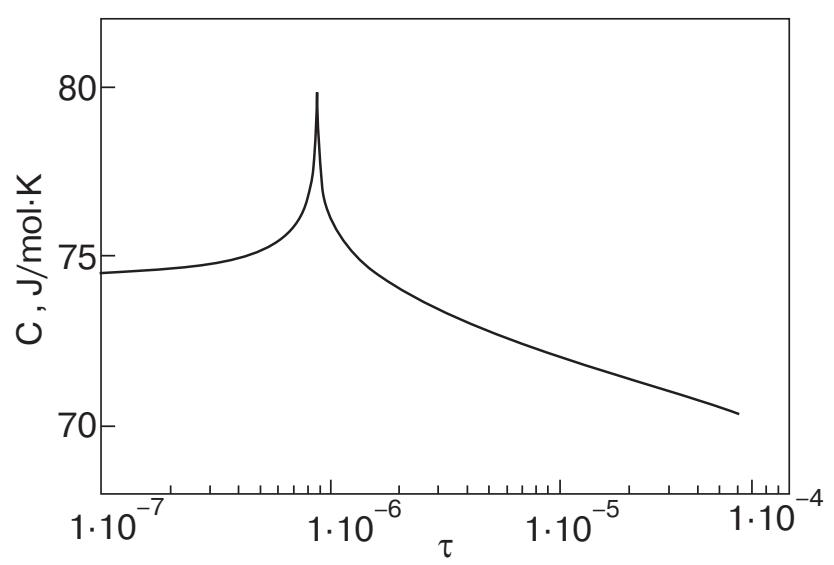

Fig. 3. The dependence of the confined helium heat capacity $C$ on the reduced temperature $\tau$ for the 8 - $\mu \mathrm{m}$ diameter cylindrical sample in semilog scale (according to Eq.(5)). 
The heat capacity of ${ }^{4} \mathrm{He}$ confined in an $8-\mu \mathrm{m}$ diameter cylindrical sample versus reduced temperature on a logarithmic scale is presented in Fig. 3. This data set, calculated from Eq. (5), obviously corresponds to the region of negative values of reduced temperature $\tau \leq 0$, where $T<T_{\lambda}$.

\section{Conclusions}

Finally, it should be noted that over the range of temperature variable from $10^{-2}$ - with nanokelvin accuracy - up to the new transition temperature of the confined system the correlation length, calculated from Eq. (3) in the same way at the heat capacity but with the corresponding critical exponent $v$, demonstrates growth at a rate of about $10^{5}$ times. Consequently, the correlation length reaches a value of the order of tens of micrometers, which in most of the cases considered cases is much greater than the diameter of the cylinder. In fact, the same set of formulas gave good agreement with the experimental results for cylindrical geometry in terms of the shift of transition temperature and also of the rate of heat capacity growth near the new transition temperature. We believe that other static and dynamical physical properties of finite-size systems, which could be represented near the criticality in terms of scaling relations with a correlation length, could be described in the framework of this study. At this point, the current theoretical approach gave results that are reasonably matched to confined ${ }^{4} \mathrm{He}$ heat capacity experimental data over a wide range of system sizes, from tens of nanometers up to about ten micrometers for the cylindrical type of geometry.

In further development of this study we are going to present the results [25] of the heat capacity calculations in the cases of planar and barlike geometry and confirm their consistency with a number of available experimental results $[1,7,8,34-36]$ and Monte Carlo simulations $[10,18]$.

The results of the complex experimental, numerical and theoretical research on confined systems are considered to be essential for fundamental studies. However, it could be even more important for providing authentic and explicit information on the static and dynamic properties of liquid matter on the micro- and nano-scales to scientists and engineers for practical applications in the field of technical problem-solving and the development of the novel technologies.

K. Ch. would like to express heartiest gratitude to deceased Prof. K. Hamano, Japan and to thank Prof. L. Bulavin at Kiev National University, Prof. A. Chalyi at National Medical University, Ukraine, professors K. Kubota and T. Yamamoto at Gunma
University, Japan for numerous useful discussions and enlightening comments in the course of this research.

1. J.A. Lipa, M. Coleman, D.R. Swanson, J.A. Nissen, Z.K. Geng, and K. Kim, Physica B280, 50 (2000).

2. G. Fasol, Science 280, 545 (1998).

3. Tar-pin Chen and F.M. Gasparini, Phys. Rev. Lett. 40, 331 (1978).

4. J.A. Lipa, D.R. Swanson, J.A. Nissen, Z.K. Geng, P.R. Williamson, D.A. Stricker, T.C.P. Chui, U.E. Israelsson, and M. Larson, Phys. Rev. Lett. 84, 4894 (2000).

5. J.A. Lipa, M. Coleman, and D.A. Stricker, J. Low Temp. Phys. 124, 443 (2001).

6. M. Coleman and J.A. Lipa, Phys. Rev. Lett. 74, 286 (1995).

7. S. Mehta and F.M. Gasparini, Phys. Rev. Lett. 78, 2596 (1997).

8. S. Mehta, M.O. Kimball, and F.M. Gasparini, J. Low Temp. Phys. 114, 467 (1999).

9. X. Qin, J.A. Nissen, D.R. Swanson, P.P. Williamson, D.A. Stricker, J.A. Lipa, T.C.P. Chui, and U.E. Israelsson, Cryogenics 36, 781 (1996).

10. N. Schultka and E. Manousakis, Phys. Rev. Lett. 75, 2710 (1995).

11. A.Z. Patashinskii and V.L.Pokrovskii, The Fluctuation Theory of Phase Transitions, Pergamon Press, Oxford, (1979).

12. M.E. Fisher, in: Critical Phenomena, Proc. Intern. School of Physics «Enrico Fermi» Course 51, M.S. Green (ed.), Academic Press, New York (1971).

13. M.E. Fisher, Rev. Mod. Phys. 46, 597 (1974).

14. L.S. Goldner and G. Ahlers, Phys. Rev. B45, 13129 (1992).

15. G. Ahlers, J. Low Temp. Phys. 113, 835 (1998).

16. G. Ahlers, J. Low Temp. Phys. 115, 143 (1999).

17. K. Nho and E. Manousakis, Phys. Rev. B64, 144513 (2001).

18. N. Schultka and E. Manousakis, J. Low Temp. Phys. 111, 783 (1998).

19. G. Ahlers, Phys. Rev. 171, 275 (1968).

20. P.B. Weichman, A.W. Harter, and D.L. Goodstein, Rev. Mod. Phys. 73, 1 (2001).

21. J.L. Lebowitz and J.L. Percus, Phys. Rev. 122, 1675 (1961).

22. K.A. Chalyi, K. Hamano, and A.V. Chalyi, J. Mol. Liquid. 92, 153 (2001).

23. V. Dohm, Phys. Script. 49, 46 (1993).

24. P. Sutterand and V. Dohm, Physica (Amsterdam) B194-196, 613 (1994).

25. K.A. Chalyy, Doctor Thesis, Gunma Univ., Japan (2001).

26. S. Bhattacharyya and J.K. Bhattacharjee, Phys. Rev. B58, 15146 (1998).

27. M.E. Fisher, Rep. Prog. Phys. 30, 615 (1967).

28. K.H. Mueller, G. Ahlers, and F. Pobell, Phys. Rev. B14, 2096 (1976).

29. J.A. Lipa, D.R. Swanson, J.A. Nissen, T.C.P. Chui, and U.E. Israelsson, Phys. Rev. Lett. 76, 944 (1996). 
30. J.A. Lipa, M.J. Buckingham, and C. Edwards, Phys. Rev. Lett. 25, 1086 (1970).

31. A. Singsaas and G. Ahlers, Phys. Rev. B30, 5103 (1984).

32. I.S. Gradstein and I.M. Ryzhik, Tables of Integrals, Sums, Rows, and Products, Nauka, Moscow (1971) (in Russian).
33. K. Binder, Annu. Rev. Phys. Chem. 43, 33 (1992).

34. M.O. Kimball, S. Mehta, and F.M. Gasparini, J. Low Temp. Phys. 121, 29 (2000).

35. S. Mehta, M.O. Kimball, and F.M. Gasparini, J. Low Temp. Phys. 113, 435 (1998).

36. J.A. Nissen, T.C.P. Chui, and J.A. Lipa, J. Low Temp. Phys. 92, 353 (1993). 